\title{
A NOTE ON A PAPER OF BOWCOCK AND YU
}

\section{I.P. STAVROULAKIS}

Consider the first order differential equation (1) $x^{\prime}(t)+\sum_{i=1}^{n} p_{i} x\left(t-\tau_{i}\right)=0$, where $p_{i}$ and $\tau_{i}$, for $i=1, \ldots, n$, are positive constants. To find necessary and sufficient conditions, in terms of the coefficients and the delays only, under which all solutions of (1) oscillate, is a problem of great importence. In a recent paper, Bowcock and Yu claimed that $\sum_{i=1}^{n} p_{i} \tau_{i}>1 / e$ is a necessary and sufficient condition for all solutions of (1) to be oscillatory. In this paper a counterexample shows that the above result is not valid and the error in this paper is indicated.

Retarded differential equations provide mathematical models for systems in which the rate of change depends not only on their present stage, but also on their past history. Such equalions appear in control theory, biology, ecology, economics, physics etcetra. Recently the study of the oscillatory behaviour of solutions of first order differential equations with retarded and advanced arguments has been a subject of great interest. See, for example, $[1-8]$ and the references cited therein. A characteristic feature of the work is that when the arguments are zero, that is, in the case of a first order ordinary differential equation, there are no oscillatory nontrivial solutions.

As is custormary, a solution is called oscillatory if it has arbitrarily large zeros. Otherwise it is called nonoscillatory.

For the differential equation with one retarded argument

$$
x^{\prime}(t)+p x(t-\tau)=0
$$

and the equation with one advanced argument

$$
x^{\prime}(t)-p x(t+\tau)=0
$$

where $p$ and $\tau$ are positive constants, the condition

$$
p \tau>\frac{1}{e}
$$

is necessary and sufficient for all solutions of $(1)$ and $\left(1^{\prime}\right)$ to oscillate (see $\left.[4,6]\right)$.

For differential equations with several arguments no such statement has been found yet. However, combining results due to Ladas and Stavroulakis [7], Arino, Gyori and Jawhari [1] and to Hunt and Yorke [3], the following sufficient conditions were obtained.

Received 20 December, 1988

Copyright Clearance Centre, Inc. Serial-fee code: 0004-9729/89 \$A2.00+0.00. 
THEOREM 1. Consider the differential equation with retarded arguments

$$
x^{\prime}(t)+\sum_{i=1}^{n} p_{i} x\left(t-\tau_{i}\right)=0
$$

and the differential equation with advanced arguments

$$
x^{\prime}(t)-\sum_{i=1}^{n} p_{i} x\left(t+\tau_{i}\right)=0
$$

where $p_{i}$ and $\tau_{i}$ for $i=1, \ldots, n$ are positive constants. Then any one of the following conditions

$$
\sum_{i=1}^{n} p_{i} \tau_{i}>\frac{1}{e}
$$

or

$$
\left[\prod_{i=1}^{n} p_{i}\right]^{1 / n}\left(\sum_{i=1}^{n} \tau_{i}\right)>\frac{1}{e}
$$

implies that all solutions of (3) and ( $\left.3^{\prime}\right)$ oscillate.

It is easily seen that the conditions (4) and (5) above are independent. They are also sharp in that the lower bound $1 / e$ cannot be improved. Finally when $n=1$, that is, in the case of one argument (4) and (5) reduce to (2).

Note that the characteristic equation of $(1)$ and $\left(1^{\prime}\right)$ is

$$
\lambda+p e^{-\lambda \tau}=0
$$

and the condition (2) is equivalent to seeing that (6) has no real roots.

A necessary and sufficient condition (in terms of the characteristic equation) for the oscillation of all solutions of $(3)$ and $\left(3^{\prime}\right)$ is given in the following theorem (see [8, $\mathbf{5}, \mathbf{1}, \mathbf{3}])$.

THEOREM 2. A necessary and sufficient condition for the solutions of (3) and (3') to oscillate is that

$$
-\lambda+\sum_{i=1}^{n} p_{i} e^{\lambda \tau_{i}}>0, \text { for all } \lambda>0
$$

or, equivalently, that the characteristic equation

$$
-\lambda+\sum_{i=1}^{n} p_{i} e^{\lambda \tau_{i}}=0
$$


of $(3)$ and $\left(3^{\prime}\right)$ has no real roots.

The advantage of working with the two conditions (4) and (5) rather than the characteristic equation (8) directly is that (4) and (5) are explicit (in terms of the coefficients and the arguments only), while determining whether or not a real solution to Equation (8) exists may be quite a problem in itself. Therefore, to find a necessary and sufficient condition, in terms of the coefficients and arguments only, for all solutions of (3) and $\left(3^{\prime}\right)$ to oscillate, is a problem of great importance.

Very recently Bowcock and Yu [2], based on [1], obtained the above condition (7) and also claimed that (Theorem 2.4 and 3.4 of $[2]$ ):

A necessary and sufficient condition for all solutions of $(3)$ and $\left(3^{\prime}\right)$ to oscillate is that

$$
\sum_{i=1}^{n} p_{i} \tau_{i}>\frac{1}{e}
$$

That is, condition (4) is not only sufficient (as is stated in Theorem 1) but also necessary. However, as the following counterexample ([7, Example 4.3]) indicates, the above result is not valid.

Example. Consider the differential equations

$$
x^{\prime}(t)+x\left(t-\frac{1}{10}\right)+\frac{1}{4} x(t-1)=0
$$

and

$$
x^{\prime}(t)-x\left(t+\frac{1}{10}\right)-\frac{1}{4} x(t+1)=0 .
$$

Observe that

$$
\left(\tau_{1}+\tau_{2}\right) \sqrt{p_{1} p_{2}}=\left(\frac{1}{10}+1\right) \sqrt{1 \frac{1}{4}}=\frac{11}{20}>\frac{1}{e},
$$

that is, condition (5) of Theorem 1 is satisfied and therefore all solutions of these equations oscillate. However

$$
p_{1} \tau_{1}+p_{2} \tau_{2}=\frac{1}{10}+\frac{1}{4}<\frac{1}{e}
$$

that is, condition (4) is not satisfied.

In fact, the proof of Theorems 2.4 and 3.4 in [2] is not correct, since the authors use the well-known inequality

$$
\min \left\{\sum_{i=1}^{n} f_{i}(x)\right\} \geqq \sum_{i=1}^{n}\left(\min f_{i}(x)\right)
$$

with the sign of equality only. That is, in their proofs inqualities (2.13) and (3.13) do not imply inequalities (2.14) and (3.14) respectively.

Therefore, the interesting problem mentioned above remains open. 
Problem. Find necessary and sufficient conditions, in terms of the coefficients and arguments only, for all solutions of Equation (3) to oscillate

\section{REFERENCES}

[1] O. Arino, I. Györi and A. Jawhari, 'Oscillation criteria in delay equations', J. Differential Equations 53 (1984), 115-123.

[2] J.E. Bowcock and Y.H. Yu, 'Sharp conditions for oscillations caused by retarded and advanced perturbations', Bull. Austral. Math. Soc. 37 (1988), 429-435.

[3] B.R. Hunt and J.A. Yorke, 'When all solutions of $x^{\prime}=-\sum q_{i}(t) x\left(t-T_{i}(t)\right)$ oscillate', J. Differential Equations 53 (1984), 139-145.

[4] G. Ladas, 'Sharp conditions for oscillations caused by delays', Appl. Anal. 9 (1979), 93-98.

[5] G. Ladas, Y.G. Sficas and I.P. Stavroulakis, 'Necessary and sufficient conditions for oscillations', Amer. Math. Monthly 80 (1983), 637-640.

[0] G. Ladas and 1.P. Stavroulakis, 'On delay differential inequalities of first order', Funkcial. Ekvac. 25 (1982), 105-113.

[7] G. Ladas and 1.P. Stavroulakis, 'Oscillations caused by several retarded and advanced arguments', J. Differential Equations 44 (1982), 134-152.

[8] M.I. Tramov, 'Conditions for oscillatory solutions of first order differential equations with delayed arguments', Izv. Vyssh. Uchebn. Zaved. Mat. 19 (1975), 92-96.

Department of Mathematics

University of Ioannina

45130 Ioanniana

Greece 